\title{
Entrelacs
}

ENTRELACS Cinéma et audiovisuel

11 | 2014

La Voix

\section{La voix lyrique à l'écran ou le rapport voix-corps dans le film d'opéra}

\author{
Laure-Hélène Sacco
}

\section{(2) OpenEdition}

Journals

Édition électronique

URL : http://journals.openedition.org/entrelacs/1565

DOI : 10.4000/entrelacs.1565

ISSN : 2261-5482

Éditeur

Éditions Téraèdre

Référence électronique

Laure-Hélène Sacco, «La voix lyrique à l'écran ou le rapport voix-corps dans le film d'opéra », Entrelacs [En ligne], 11 | 2014, mis en ligne le 14 janvier 2015, consulté le 30 avril 2019. URL : http:// journals.openedition.org/entrelacs/1565; DOI : 10.4000/entrelacs.1565

Ce document a été généré automatiquement le 30 avril 2019

Tous droits réservés 


\title{
La voix lyrique à l'écran ou le rapport voix-corps dans le film d'opéra
}

\author{
Laure-Hélène Sacco
}

1 Le film d'opéra correspond à la rencontre de deux formes d'art, de deux esthétiques. Soumettre l'art lyrique à une écriture cinématographique engendre des répercutions techniques et musicales au niveau de l'adaptation, de l'interprétation et de la réalisation. L'objectif de cette étude est de traiter tout particulièrement la question du passage à l'écran de la voix lyrique et de s'interroger sur la problématique qu'il soulève sur le plan du rapport voix-corps. Il s'agit de se demander, pour une adaptation cinématographique d'un opéra, comment appréhender la voix chantée, comment filmer le corps chantant, tout en conciliant les exigences du cinéma et celles de l'opéra.

Pour comprendre comment il est possible de faire coïncider tant esthétiquement que techniquement le corps et la voix à l'écran, nous aborderons la question du doublage et du play-back, les inconvénients et les avantages qu'ils présentent. Il s'agira d'évaluer l'enjeu de ce dédoublement entre la voix et le corps sur les plans de la synchronisation mouvements-voix et de la vraisemblance de la gestuelle des interprètes. Cette étude vise aussi à mesurer l'impact de ce passage à l'écran sur le travail vocal, corporel et scénique du chanteur lyrique, ainsi que la façon dont il l'adapte à l'écriture et à l'image cinématographique.

Plus précisément, après avoir défini la gageure du doublage, nous analyserons l'articulation de la technique du play-back et des problématiques liées au corps, aux apparences et aux capacités physiques du chanteur. Nous nous interrogerons par la suite sur la manière d'appréhender techniquement et esthétiquement le play-back, en abordant le travail de synchronisation, le mimétisme et les choix relatifs à la monstration du chant. Nous apprécierons alors la manière dont les cinéastes se sont accommodés de cette dissociation due au play-back, puis nous verrons que si elle apparait parfois comme un obstacle, elle peut aussi s'avérer porteuse de libertés créatives. Enfin, nous nous intéresserons à la question de la réception, en analysant la façon dont ont été reçus par la 
critique ces choix relatifs au rapport voix-corps dans le film d'opéra et plus exactement à l'emploi du play-back.

Pour étayer l'ensemble de cette réflexion, nous axerons notre étude sur quatre films d'opéra : Don Giovanni de Joseph Losey (1979), Carmen de Francesco Rosi (1984), la Bohème de Luigi Comencini (1987), et Tosca de Benoît Jacquot (2000).

\section{La gageure du doublage : faire coïncider crédibilité physique et crédibilité du chant}

4 Le cinéma permettant des plans rapprochés, le chanteur d'un film d'opéra doit posséder, en plus d'un jeu d'acteur affiné et d'une technique vocale aboutie, un physique un minimum en adéquation avec celui que présuppose le rôle, malgré un recours possible au maquillage et aux effets spéciaux (cas des productions les plus récentes) pour le modifier. Ces trois conditions étant difficiles à réunir, le réalisateur a la plupart du temps recours à la technique du play-back, qui permet une dissociation entre la voix et le visage, entre le son et l'image. Il est alors libre de décider que le chanteur responsable de l'enregistrement sonore interprète également le rôle à l'écran ou opter pour un jeu de doublage lui permettant de prêter la voix du chanteur à un autre acteur, dont le physique et l'âge correspondent mieux au rôle.

5 Cette technique était surtout répandue pour les films d'opéra les plus anciens. Nous pouvons citer l'exemple du Rigoletto de Carmine Gallone datant de 1946, pour lequel le réalisateur a employé la technique du dédoublement acteur-chanteur afin de répondre aux canons de beauté de l'époque, ainsi qu'aux exigences du rôle de Gilda. La chanteuse Lina Pagliughi double en effet la jeune Marcella Govoni dont les traits purs, délicats et candides correspondent davantage à ceux du personnage.

Dans la Bohème, le rôle du jeune Rodolfo est interprété à l'écran par Luca Canonici mais est chanté par José Carreras. Toutefois, dans ce cas précis, ce dédoublement ne relève pas d'un choix artistique de la part du réalisateur. En effet, il était initialement prévu que le célèbre ténor espagnol interprète le rôle à la fois à l'écran et sur la bande-son. Or il tomba gravement malade et dut abandonner le projet alors même que l'enregistrement avait déjà été effectué et le tournage, entamé.

7 Si cette technique de doublage permet de choisir un acteur au physique plus adéquat, elle présente néanmoins des inconvénients relatifs à la synchronisation, à la vraisemblance du rapport gestuelle-voix. De fait, adopter les attitudes d'un chanteur pour un acteur est un travail ardu, car, comme le souligne le journaliste Michel Sarazin, «le maintien des épaules, la respiration, l'articulation d'un comédien ne sont pas celles d'un chanteur ${ }^{1}$. Il peut donc être difficile pour certains comédiens de s'approprier les postures et les mimiques d'un chanteur, de faire coïncider voix et visage, ce qui rend parfois le play-back peu crédible. Tel est le cas, dans le Rigoletto précédemment cité, de l'actrice Marcella Govoni, qui conserve invariablement un visage hiératique, y compris lors des montées en puissance ou dans les aigus, lesquelles devraient au contraire causer une distorsion du visage. En effet, l'exploitation du registre aigu de la tessiture en chant lyrique sollicite d'autant plus les muscles du visage, en particulier ceux du «masque», c'est-à-dire la région correspondant aux résonateurs, ce qui peut par exemple entrainer une surélévation des pommettes, en tout cas le signe manifeste d'un effort corporel, perceptible aussi dans le cou ou l'ensemble de la posture. Plus spécifiquement, chanter 
des notes suraiguës, c'est-à-dire atteindre l'extrémité haute de la tessiture, peut aussi nécessiter une ouverture plus importance de la bouche. Ainsi, là où l'on s'attend à percevoir les marques d'un effort physique, il paraît surprenant qu'un visage conserve les mêmes expressions que lors d'un discours parlé.

Pour ces différentes raisons, les réalisateurs de films d'opéra ont très majoritairement souhaité que les chanteurs incarnent aussi leur rôle à l'écran. De plus, la dimension vocale étant l'essence de l'opéra, ils se sont même montrés soucieux, bien souvent, de représenter avec réalisme l'exercice du chant, point sur lequel nous reviendrons. Choisir un chanteur pour interpréter intégralement le rôle peut donc aussi être une façon de respecter la logique même du film d'opéra. Puisqu'il s'agit avant tout de musique, il paraissait normal à Francesco Rosi de confier les rôles à des musiciens ${ }^{2}$. Quitte à travailler avec des comédiens, se justifiait-il, il aurait filmé la Carmen de Mérimée, selon son projet initial. L'intérêt de cette adaptation de Bizet résidait donc justement dans cette rencontre entre le monde de l'opéra, celui des chanteurs lyriques et celui du cinéma.

Luigi Comencini partageait ce point de vue. Comme évoqué précédemment, le doublage à l'écran de José Carreras était indépendant de sa volonté. Ce fut la seule solution possible suite au départ précipité du ténor espagnol, puisque l'enregistrement musical avait déjà été achevé. Cependant, le réalisateur avait mis un point d'honneur à ce que son remplaçant soit lui aussi un chanteur. «Un chanteur n'articule pas, ne respire pas comme un comédien et cela aurait pu nuire au synchronisme son-image $»^{3}$, rappelle Jacques Siclier dans le journal Le Monde, à la sortie du film. Ce choix a donc été effectué dans un souci de crédibilité et de réalisme dans la gestuelle.

10 Ainsi, le doublage, qu'il soit assuré par un comédien distinct du chanteur ou par le chanteur lui-même, nécessite, pour des raisons de vraisemblance, de faire coïncider crédibilité physique et crédibilité du chant, problématique que nous allons maintenant développer en deux temps.

\section{Le play-back et les problématiques liées au corps, aux apparences et aux capacités physiques du chanteur}

11 Si le fait de faire jouer au chanteur le rôle qu'il a enregistré musicalement facilite la crédibilité de sa gestuelle à l'image, il pose néanmoins le problème de l'adéquation entre le physique de l'interprète et celui de son personnage : le cinéma pouvant proposer une forme de narration plus intime, celle-ci est bien plus importante qu'à la scène. En salle, le spectateur ne peut avoir qu'une vision d'ensemble et plutôt lointaine de la représentation. C'est donc la voix qui prédomine. Le chanteur peut ainsi se contenter de n'avoir que l'allure de son personnage, à défaut d'en avoir précisément le physique. Le cinéma, lui, propose une proximité plus grande avec les personnages et appelle donc à une exigence accrue quant à l'apparence physique. Le talent dramatique d'un chanteur d'opéra ne lui suffit donc pas pour être un bon acteur de cinéma. Son jeu de scène peut très bien être convaincant au théâtre et perdre de sa crédibilité à l'écran. En dépit des subterfuges rendus possibles par le maquillage ou la post-production, il lui faut de préférence posséder un physique proche de celui du personnage, ainsi que cette compatibilité instinctive avec la caméra. D'où la difficulté des cinéastes à trouver un chanteur ou une chanteuse qui présente non seulement les capacités vocales requises, mais aussi ces qualités physiques et dramatiques. Aussi, pour atteindre cette adéquation 
entre voix, visage et âge, il a parfois fallu aux chanteurs effectuer tout un travail sur leur corps, différent de celui effectué pour la scène, afin d'accroître la crédibilité physique de leur personnage. Dans le cas de Don Giovanni, Frantz Salieri, scénariste et costumier du film, a dû effectuer un réel travail de métamorphose sur Ruggero Raimondi, qui incarne le premier rôle. Il a joué minutieusement sur le maquillage, les costumes, les perruques pour lui donner l'allure d'un véritable Don Juan. Le travail de Frantz Salieri a dû aussi s'accompagner d'un profond investissement personnel de la part du chanteur : pour se fondre pleinement dans le corps du séducteur, celui-ci a dû suivre tout un cheminement psychologique, et parfois même céder à certains sacrifices pour accepter des modifications physiques, qui devenaient nécessaire pour un passage devant la caméra. Par exemple, Frantz Salieri estimait que sa barbe noire, qui obscurcirait trop son visage, était problématique. Il eut donc à lui expliquer « la difficulté de faire, avec cette barbe, une approche sérieuse du visage qu'il porterait dans l'exercice du rôle $»^{4}$. Cependant, Ruggero Raimondi donnait un concert quelques jours plus tard et demanda à son costumier de laisser passer cette échéance pour sacrifier sa barbe, tant associée alors à son image de soliste. Frantz Salieri avouait comprendre "sa répugnance pour une nudité soudaine devant un public accoutumé à une autre apparence et qui le livrerait sans masque, aussitôt après, à une expérience qu'il redoutait $»^{5}$. Il fut donc étonné de le voir arriver rasé dès le lendemain, pour les premiers essais filmés : «j'admirais qu'un homme méprisât son confort et quelque chose de plus pour être exactement celui qu'il fallait qu'il soit même quand il s'agissait d'une obligation sans urgence et désagréable " ${ }^{6}$, affirmait-il. Pour son rôle d'Escamillo dans la Carmen de Francesco Rosi, Ruggero Raimondi aurait par ailleurs volontairement perdu une dizaine de kilos pour devenir un torero crédible et séduisant, et Placido Domingo, une vingtaine, pour avoir, sous les traits de Don José, l'apparence d'un soldat vigoureux et attirant, là où une représentation en salle requerrait peut-être moins de rigueur quant à la morphologie du personnage et donc de son interprète. Luca Canonici a lui été choisi dans la Bohème pour sa ressemblance physique avec José Carreras. Cependant, un problème persistait : l'adéquation entre l'âge de ce nouveau Rodolfo et celui de la voix enregistrée. Luca Canonici était trop jeune pour donner l'impression qu'il s'agissait vraisemblablement de sa voix. José Carreras était non seulement plus âgé, mais aussi plus expérimenté : sa carrière était déjà bien entamée, et sa voix, plus ample, plus travaillée. Il risquait donc d'y avoir une dissonance dans le rapport voix-visage. C'est pourquoi Luca Canonici a dû lui aussi céder à une transformation physique et s'est vu vieilli par l'ajout de quelques cheveux blancs'.

En raison de cette difficulté de faire coïncider exigences physiques et exigences vocales, Francesco Rosi eut de la peine à dénicher sa Carmen car il tenait à ce que sa chanteuse, en plus d'avoir le caractère et le physique du rôle, présente des talents de danseuse. En effet, dans cet opéra, la danse influence considérablement la musique de Bizet et caractérise l'univers du personnage principal. C'est la raison pour laquelle Francesco Rosi a choisi d'accorder une place importante à la dimension chorégraphiée dans son film et de faire écho à la musique de Bizet inspirée de danses populaires. Julia Migenes-Johnson se situait en l'occurrence au confluent de toutes les exigences requises par Francesco Rosi pour le rôle de Carmen. Elle semblait notamment répondre aux critères physiques souhaités par Francesco Rosi qui s'inspiraient des croquis de Mérimée ou encore de Gustave Doré. Le cinéaste avait en effet trouvé en cette chanteuse une réplique parfaite des gitanes représentées sur ces esquisses aux "traits physiques forts ", à la "beauté qui vient de l'intérieur $»^{9}$. En revanche, elle présentait l'inconvénient d'une part d'être soprano alors 
que le rôle est celui d'une mezzo, de l'autre, de manquer de puissance et de largeur par rapport au profil vocal du personnage. Si elle n'a donc pas eu à changer grandement son apparence physique, il a en revanche fallu à Julia Migenes-Johnson modifier sa voix, travailler ses graves et modeler son timbre pour avoisiner le registre et la couleur vocale d'une mezzo ${ }^{10}$. Aussi, l'enregistrement musical ayant lieu en studio et non en direct, celuici peut se faire de façon fragmentée, autorisant ainsi des pauses. L'interprète n'est donc pas amené à filer l'œuvre, comme il doit le faire sur scène. De la sorte, le play-back permet de faire intervenir un chanteur ou une chanteuse qui, pour des raisons d'aisance, de force ou d'endurance, n'aurait pu interpréter le rôle à la scène : Julia Migenes-Johnson reconnaissait elle-même ne pas pouvoir assumer sur scène ce rôle de Carmen, qui n'était pas dans sa tessiture. Elle avouait manquer de puissance au point qu'il lui aurait été difficile de couvrir musicalement un orchestre. Ses graves, principalement, n'auraient pas été assez sonores pour dépasser la fosse. Au cinéma, il est en revanche possible de pallier ces problèmes d'intensité en jouant, en post-production, sur les niveaux sonores grâce aux techniques d'enregistrement.

Par ailleurs, le play-back présente l'avantage de pouvoir dissocier le travail vocal de l'interprétation dramatique. Ainsi, le fait d'enregistrer préalablement leur prestation musicale permet aux chanteurs de se concentrer pleinement sur leur performance vocale lors de l'enregistrement, et sur leur jeu d'acteur au moment du tournage. Ils ont en effet la possibilité de chanter à mi-voix lors du tournage qui s'effectue au son de l'enregistrement ${ }^{11}$. Cela leur permet donc d'être plus endurants.

En outre, à l'écran, les personnages sont nécessairement plus mobiles qu'à la scène, de façon à éviter toute fixité de l'image. Dans le cadre d'un spectacle vivant et en direct, les possibilités, pour les chanteurs, de se mouvoir en chantant sont plus restreintes, pour des raisons évidentes de performance physique. C'est justement cette dissociation de la voix et du corps qui a permis à Francesco Rosi d'accorder dans son film cette place toute particulière à la danse : libérés de leur interprétation vocale au moment du tournage, les chanteurs acquièrent davantage de liberté dans leur expression corporelle. De cette façon, Julia Migenes-Johnson a pu offrir à sa Carmen une gestuelle exubérante, lascive et très dansante.

Selon Rolf Liebermann, collaborateur artistique du Don Giovanni de Joseph Losey, une captation du son en direct aurait de toute façon été trop complexe, aussi bien d'un point de vue technique que pour des raisons liées au travail des chanteurs. En effet, sur un plateau de tournage, ces derniers ne peuvent avoir la même endurance que des comédiens. À ses yeux, le play-back était en ce sens «un mal nécessaire, car il est impossible de demander à un chanteur de chanter pendant une heure pour une prise d'une minute, comme cela se fait sans problème avec les acteurs $»^{12}$. C'est pourquoi il évoquait l' « impossibilité physique et matérielle » d'envisager un enregistrement sur le vif, concomitant au tournage.

\section{Appréhender techniquement et esthétiquement le play-back : le travail de synchronisation, le mimétisme et les choix relatifs à la monstration du chant}

$\mathrm{Au}$ vu des multiples contraintes techniques qu'elle implique, une captation du son direct s'avère très coûteuse et peut-être même assez restrictive d'un point de vue créatifi ${ }^{13}$. 
Aussi, est-ce une des raisons pour lesquelles les réalisateurs ont très majoritairement opté pour le play-back. Toutefois, même lorsque les chanteurs doublent leur propre voix, cette technique pose le problème d'une difficile synchronisation labiale, comme l'indiquent leurs différents témoignages. Par exemple, Barbara Hendricks, l'interprète de Mimì dans la Bohème de Luigi Comencini, évoquait l'exigence de ce travail de précision qui demande "d'être fidèle au millième de seconde près à ce qu'on a préalablement enregistré $»^{14}$, et qui requiert un long travail préliminaire: "Chaque matin, tous les chanteurs devaient répéter le play-back de l'après-midi avec un assistant spécialisé. Au cinéma, il faut penser d'abord, et les lèvres suivent. Sur scène, c'est le contraire ${ }^{15}$. Ruggero Raimondi admettait quant à lui que le play-back permettait de rendre le tournage moins fatigant, mais il avouait surtout la complexité d'être synchrone ${ }^{16}$. Outre le fait d'être exactement en mesure, il expliquait que cette technique impliquait «[de] se plonger complètement dans la musique, et dans cette limite, jouer la comédie, sans public, devant une caméra qu'on doit regarder avec passion $»^{17}$. La difficulté principale était à ses yeux de trouver sa liberté dans ce cadre prédéfini, tout en admettant qu'au bout d'un certain temps, il pouvait devenir une aide et baliser leur travail ${ }^{18}$. Dans le cas du Don Giovanni de Joseph Losey, la responsable de la synchronisation était Janine Reiss, également claveciniste, chef de chant et répétitrice musicale sur le tournage. Cette tâche la terrorisait car elle se sentait investie d'un devoir d'excellence, alors qu'il lui était particulièrement difficile de veiller à cette coordination durant les ensembles où tous chantaient en même temps ${ }^{19}$. Pour Luca Canonici dans la Bohème, la difficulté du play-back a été accrue par le fait qu'il doublait une autre voix que la sienne, et, qui plus est, celle d'un des ténors les plus reconnus au monde. Il sentait lui aussi en charge d'une lourde responsabilitée ${ }^{20}$. Sa mission fut d'autant plus périlleuse que le remplacement de José Carreras avait dû se faire précipitamment et qu'il ne connaissait pas le rôle au préalable.

17 En outre, certains chanteurs, comme Barbara Hendricks, se sont avoués troublés par la méthode du play-back qui, contrairement à une représentation en salle, fait perdre l'immédiateté de la transmission du chant et de l'émotion. En ce sens, cette dissociation a eu une incidence sur leur interprétation, sans doute parce que l'échange direct avec le public et l'énergie qu'il transmet leur ont fait défaut ${ }^{21}$.

18 Le passage à l'écran implique par ailleurs pour le chanteur une modification de son jeu théâtral et de sa pratique vocale, en particulier parce que les points de vue et les espaces scéniques se multiplient, se diversifient. Cela exige donc de lui qu'il adapte ses mouvements, sa gestuelle et son interprétation à la caméra et à ce nouvel espace de jeu. Il lui faut rompre avec ses habitudes scéniques et vocales en cherchant un moyen d'expression nouveau. Les chanteurs qui nous intéressent sont, pour la plupart, de réelles « vedettes » de la scène lyrique. Ils sont, par conséquent, habitués aux plus grandes salles d'opéra du monde. Lors des représentations, la distance qui les sépare du public est donc assez importante. Devant la caméra, ils sont contraints d'adapter leur façon de chanter et de jouer aux points de vue plus intimistes du cinéma qui soulignent chacun de leurs gestes et de leurs expressions. Leurs témoignages nous éclairent sur la façon dont s'opère cette transition entre la scène et l'écran, et ce qu'elle implique physiquement chez ces chanteurs. D'une manière générale, tous s'accordent pour dire que la principale difficulté réside dans la diminution de la distance espace scénique-point de réception, car elle les oblige à réviser intégralement leur façon de jouer. Sur scène, ils doivent faire des gestes amples pour accroître l'emphase lyrique et être suffisamment expressifs afin d'être vus de loin. Au cinéma, en revanche, ils doivent opter pour une gestuelle plus naturelle, une 
interprétation plus intériorisée et authentique pour éviter de sur-jouer. En salle, l'exagération est estompée par la distance. À l'écran, les cadrages plus resserrés ne feraient que grossir le trait d'un tel jeu, qui en deviendrait grandiloquent, voire ridicule. Ruggero Raimondi évoquait la nécessité d'une direction d'acteur précise, destinée à guider le chanteur vers une interprétation plus profonde, plus canalisée ${ }^{22}$. Pour éviter tout risque de caricature, Joseph Losey rappelait l'importance, pour les chanteurs, de se montrer économes de leurs mouvements lorsqu'ils passent devant la caméra ${ }^{23}$. Lors des premiers essais de Don Giovanni, Ruggero Raimondi s'en est trouvé quelque peu déboussolé. Il avait confié à Frantz Salieri sa crainte de ne pas savoir trouver la juste mesure, de demeurer toujours dans l'excès ou au contraire, dans la platitude ${ }^{24}$. Forts de leur échange, les deux hommes avaient convenu que Ruggero Raimondi devrait passer outre les conventions opératiques pour laisser émerger ses émotions les plus profondes, qu'il lui faudrait « concentrer l'expression dans une gestuelle extrêmement réservée qui ne laisserait paraître qu'une sorte d'écho des tumultes intérieurs, aussi déchaînés soientils ». «À la caméra de scruter cet écho et de le ramener à la dimension de la musique »"25, lui avait affirmé Frantz Salieri. Conscient du travail qu'avait dû accomplir Ruggero Raimondi, en particulier sur son corps, sa gestuelle et ses attitudes physiques pour devenir acteur de cinéma, Frantz Salieri fut admiratif du résultat et de la justesse de son jeu. Devant la caméra, le chanteur a en effet dû jouer avec davantage de précision, comme il l'exprimait lui-même : « Le cinéma permet un approfondissement de chaque détail et le rapprochement oblige à un jeu moins global qu'au théâtre, plus fouillé. Chaque plan est étudié, analysé à fond $»^{26}$. Ainsi cette proximité et cette méticulosité de l'image l'ont-elles amené à modifier ses habitudes scéniques. Ruggero Raimondi s'était lui-même aperçu $a$ posteriori, que ce qui, dans son jeu, lui paraissait insignifiant sur scène s'avérait amplifié à l'écran.

19 En écho avec ce jeu de scène plus modéré, plus naturel, les chanteurs ont parfois dû aussi aborder le chant sous un angle plus intimiste. Les réalisateurs ont eu une approche, une conception différente du mimétisme et de la monstration du chant à l'écran. Ainsi n'ontils pas tous employé le play-back de la même façon. Cette technique leur a permis de jauger, à l'écran, l'intensité de la représentation de l'effort physique chez le chanteur.

Joseph Losey a choisi d'effectuer une prise de son directe lors des récitatifs pour un effet de réel plus fort. Pour cela, un clavecin était placé hors-champ et accompagnait les chanteurs. Il lui paraissait impensable d'adopter la même démarche pour les ensembles et les arias, ne serait-ce parce qu'ils nécessitent l'accompagnement d'un orchestre, impossible à déplacer sur un plateau de tournage en plein air. Il a donc tourné tout le reste de l'opéra en play-back. Néanmoins, il a tenu à ce que le travail physique du chanteur soit restitué à l'image, à condition d'en gommer toute exagération. Son objectif était de conserver le naturel et la réalité de l'exercice du chant, «mais pas au point de déformer la physionomie des chanteurs, de les enlaidir ou de les ridiculiser $\aleph^{27}$. Francesco Rosi, quant à lui, acceptait volontiers les distorsions du visage des chanteurs dans la mesure où il utilisait le chant comme un langage, une langue à part entière. Dans l'affrontement final entre Don José et Carmen, par exemple, il traitait le chant « comme un cri dans une discussion ou dans un combat entre deux personnes ${ }^{28}$. C'est la raison pour laquelle il ne voyait aucune raison «d'atténuer la violence de la confrontation " «Par contre, à d'autres moments, précisait-il, il faut faire attention quand il n'y a pas de justification dramatique $»^{30}$. Joseph Losey et Francesco Rosi semblaient donc en quête d'une juste mesure entre effort naturel et effort artificiel dans le mimétisme du chant: 
montrer la déformation du visage sous le coup de l'effort et la traduction physique de l'expression de la voix leur était important, car gage de sincérité et de véracité, il leur était en revanche nécessaire de se débarrasser de toute forme d'exacerbation que recherche parfois le chanteur sur scène pour être vu à distance. Ainsi, au moment du tournage en play-back, les chanteurs ont donc dû veiller à supprimer toute grimace artificielle. Julia Migenes-Johnson, par exemple, évitait d'ouvrir trop grand la bouche dès que cela apparaissait excessif et cherchait à maîtriser le moindre pas, le moindre geste ${ }^{31}$. Pour Barbara Hendricks, dirigée par Luigi Comencini dans la Bohème, ces mimiques superflues font partie de la convention opératique et il convient de s'en débarrasser face à la caméra : selon elle, «l'art lyrique, qui n'est souvent que grimaces apprises, doit y remonter à sa source. Car la convention en gros plan est insupportable $\aleph^{32}$.

Benoît Jacquot, lui, voulait initialement réaliser l'ensemble de Tosca en son direct par souci de vérité33. Il avait envie "de faire ressentir ce qui [le] subjuguait: l'intensité physique du chant, la prouesse vocale, ce sport de très haut niveau, disons, où les interprètes sont comme les cascadeurs d'eux-mêmes, parfois $»^{34}$. Forcé de tourner en play-back, il a été obligé de repenser toute sa stratégie de mise en scène. Il a cependant fini par tourner cette contrainte à son avantage, considérant "que c'est face aux impossibilités qu'on trouve ce qu'il y a de plus intéressant" ${ }^{35}$. Ainsi a-t-il estimé $a$ posteriori que le fait d'avoir été privé de son direct a finalement été une chance. Il expliquait: "J'ai dû remplacer l'authentification directe de la présence vocale par une recherche beaucoup plus élaborée et, je crois, beaucoup plus riche, mettant en évidence tous les points de frottement entre l'artifice extrême et la réalité intense de ce que je filmais $»^{36}$. Benoît Jacquot a en effet pris le parti de ne pas chercher la vérité du chant dans une synchronisation labiale parfaite, mais à travers un regard quasi documentaire sur le travail du chant en train de se faire. Il a introduit, dans son film, des séquences en noir et blanc filmées pendant l'enregistrement de la bande sonore en studio. De la sorte, la crédibilité de l'acteur chantant, ainsi acquise et démontrée par ces scènes, en donne à la fiction. N'ayant d'autre choix que de recourir au play-back, il a préféré utiliser cette technique à "contre-emploi ", c'est-à-dire en exacerbant la dissociation son-image, plutôt que de tenter de créer l'illusion, de prétendre à une excellente synchronisation qui, de toute façon, n'aurait pu égaler une prestation et une captation en direct. À ses yeux, les chanteurs, novices en matière de cinéma, n'allaient pouvoir devenir acteurs que s'ils étaient filmés dans l'exercice de leur art, c'est-à-dire lorsque, maîtrisant leur interprétation, ils étaient pleinement en mesure de livrer des émotions sincères : « $\mathrm{Si}$ j'arrivais à montrer de manière presque documentaire, concrète, ce qu'est le fait de chanter physiquement pour eux, peut-être que quelque chose de l'ordre du cinéma adviendrait dans le film $»^{37}$. De là est née cette idée des séquences documentaires autour de l'enregistrement musical. Il voulait que " la voix reste au cœur de [ses] partis pris de mise en scène » et a choisi de la " donner à "voir" dans tous ses états " ${ }^{38}$. Il expliquait en effet que «le chant, dans le film, n'est pas traité comme un état naturel, il est aussi confronté au silence, à la parole, au monologue intérieur ${ }^{39}$. Il a en effet joué sur des passages en voix parlée et a également introduit des moments de silence au cours desquels les acteurs s'arrêtent de chanter à l'écran, tandis que le play-back continue sur la bande-son, invitant les spectateurs à fondre dans les pensées intimes des personnages. Par exemple, dans l'ultime duo Tosca-Mario du troisième acte, le chant se poursuit alors que les personnages demeurent bouche bée, comme s'il s'agissait de leurs voix intérieures. Le même procédé est employé par Francesco Rosi dans Carmen, à la fin de l'air 
de Micaëla «Je dis que rien ne m'épouvante ». Cela se justifie par le fait qu'il s'agit d'une prière, d'un recueillement, c'est-à-dire d'un moment introspectif très intime.

\section{La réception par la critique des choix relatifs au rapport voix-corps et à l'emploi du play-back}

Le problème du rapport voix-visage, et en particulier celui du play-back, qui a embarrassé de nombreux cinéastes, est certainement celui qui suscite le plus de réserves auprès de la critique. Pour certains, il va jusqu'à remettre en question le principe même du film opéra, considérant le play-back comme un obstacle rédhibitoire.

Généralement, l'emploi du play-back est assez mal reçu car, en dépit de sa qualité d'exécution et des prouesses techniques qu'il démontre, ce procédé n'atteint jamais la perfection : nous pouvons parfois avoir la désagréable sensation de « voir les ficelles » du processus de création, de trop ressentir l'aspect falsifié du chant mimé. L'artificialité du play-back est souvent perçue non seulement comme un élément rompant le réalisme cinématographique de l'œuvre, mais aussi comme un obstacle émotionnel. On pourrait en ce sens parler d'un double hiatus, tant technique qu'émotionnel. Un des principaux reproches faits au play-back concerne la distance et le décalage émotionnel qu'il engendre entre le jeu du chanteur et ce qu'exprime sa voix : malgré un jeu d'acteur affiné et un mimétisme minutieux, le jeu semble toujours en retrait par rapport à la voix sur le plan de l'intensité. Étant donné qu'il ne se situe pas dans l'immédiateté de l'exercice de son art, il n'apparaît pas aussi impliqué, aussi possédé par cet effort physique que lorsqu'il chante en direct. La prise de risque liée à l'exploit vocal et la transmission de l'émotion sont moindres : devant la caméra, le chanteur n'est pas habité vocalement par son personnage comme il peut l'être au moment de l'enregistrement. Aussi convaincante soit son interprétation, l'interprète ne chante pas à l'écran. Il mime son chant. Son pouvoir expressif serait donc forcément plus limité. Pour Alain Lompech, du journal Le Monde, « un chanteur qui mime son art n'investit ni physiquement ni psychologiquement son personnage", ce qui "pasteurise les émotions" et "met musicalement hors de course les films d'opéra produits par Daniel Toscan du Plantier $»^{40}$, dont font partie les quatre films de notre corpus.

En dépit d'un travail de doublage approfondi, il peut subsister un décalage entre le son et le mouvement des lèvres du chanteur, entre la puissance émotionnelle de sa voix et l'expression de son visage ou de son corps. Ainsi le play-back est-il accusé de casser l'effet de réel du film : ces multiples imperfections fausseraient la représentation du chant, et en modifieraient donc la réception. En effet, l'enregistrement préalable, la technique de synchronisation au moment du tournage et du mixage, etc., sont autant d'obstacles à la transmission du chant, et donc de l'émotion. Il est parfois considéré que l'emploi de ces intermédiaires dénature l'essence même du chant lyrique qui, à l'origine, est destiné au spectacle vivant, et ne se prête ni au recours au micro ni à celui du play-back. L'utilisation de cette technique ferait perdre une partie de sa crédibilité au film, créant un effet de contraste dissonant entre la mise en scène qui inscrit l'action dans le réel et l'artificialité du chant. À l'occasion de la sortie de la Carmen de Francesco Rosi, le journaliste François Chalais a par exemple blâmé le fait que dans ce film qui se veut plutôt réaliste, la "perfection dans la reconstitution de l'environnement et de l'ambiance n'en [rende] malheureusement que plus sensible le décalage qui existe entre la simplicité de la réalité 
et les techniques amphigouriques de l'opéra $»^{41}:$ «On était pris par le naturel des choses de la vie, et voici que des robots ouvrent la bouche pour lancer des notes dont on voit mal le rapport qu'elles ont avec ce que l'on a si bien décrit jusque là ", affirme-t-il. Pour lui, «c'est interdire à coup sûr la montée de toute émotion $»^{42}$. À noter que François Chalais nuance tout de même son propos en ajoutant que la sincérité du jeu de Julia MigenesJohnson compense cependant la fausseté du chant engendré par le play-back.

Le recours à ce procédé, nous l'avons vu, permet au réalisateur de faire prévaloir, s'il le souhaite, les qualités physiques d'un chanteur sur ses qualités vocales. Globalement, cette démarche, qui s'inscrit en faveur d'une adaptation cinématographique réaliste, est plutôt bien reçue car elle ne fait qu'accroitre la crédibilité des personnages à l'écran. La caméra et les plans rapprochés ne feraient d'ailleurs que souligner un éventuel décalage entre l'âge de l'interprète et celui de son personnage. Ainsi la recherche de cette adéquation physique n'est-elle pas remise en cause d'un point de vue cinématographique. En revanche, cette problématique est davantage controversée sur le plan musical. Certains estiment que le fait de reléguer la dimension vocale et musicale au second rang relève du sacrilège, un opéra étant avant tout composé de musique et de chant. Pour s'appuyer sur des exemples précis, notons que Julia Migenes-Johnson et Barbara Hendricks font l'objet de nombreuses critiques à ce sujet: si on leur reconnait des ressemblances physiques évidentes avec leur personnage, elles sont souvent jugées peu compatibles vocalement avec eux. La tessiture de soprano de la première n'est pas appropriée au rôle de mezzo qu'elle occupe. Le profil de son personnage, Carmen, appelle à une couleur de voix plus ronde, plus chaude et sensuelle, avec davantage d'harmoniques graves. La seconde est souvent reconnue comme une soprano trop légère pour le rôle de Mimì, qui se prête davantage à des voix lyriques. À l'inverse, Francesco Rosi a notamment choisi Ruggero Raimondi et Placido Domingo pour la solidité de leur expérience vocale. Cependant, ce choix n'a pas fait l'unanimité, les deux chanteurs n'ayant pas réellement l'âge de leur personnage. Sur ce point, le réalisateur a été accusé de ne pas avoir choisi entre vérité et convention, de n'avoir pas suivi la même logique que pour le choix de Julia MigenesJohnson ${ }^{43}$.

Dans le cas de Tosca, les critiques ont été moins virulentes au sujet du traitement du playback. Cela tient au fait que le cinéaste n'a pas cherché à atteindre la perfection ou le réalisme de la synchronisation. De toute façon, pour certains, cela est impossible, et refuser d'assumer ce caractère factice ne fait «qu'accuser la convention des situations, des personnages et de l'intrigue $»^{44}$, pour reprendre les termes de Pascal Mérigeau du Monde. Benoît Jacquot, lui, a choisi d'assumer l'artificialité de ce procédé. Nous l'avons évoqué, il a cherché la vérité de l'exercice du chant dans les séquences tournées en studio lors de l'enregistrement, et pour les scènes de fiction, il est allé jusqu'à jouer sur les registres de voix, sans volonté particulière d'accéder à une représentation réaliste du chant. Il a donc été félicité d'avoir su transformer les inconvénients du play-back en une source de création originale ${ }^{45}$. Le fait d'exacerber cette scission entre la voix et le corps lui a permis de s'interroger, au travers de son film, sur le processus même de la création, sur la pratique de l'exercice du chant. Cette distanciation s'est vue globalement saluée par la critique. On lui a reconnu, à travers cette prise de distance, cette capacité à s'approprier les conventions du chant lyrique tout en les respectant, et à se détacher de ce carcan ${ }^{46}$. Quelques réserves ont néanmoins été émises face à tant d'originalité. réside dans une correspondance et une harmonie tant visuelles qu'auditives entre le 
corps et la voix. Le recours nécessaire au play-back, qui s'explique par l'extrême complexité et le coût exorbitant d'une captation en direct de la voix chantée, requiert en premier lieu un difficile travail de mimétisme et de synchronisation pour rendre le rapport gestuelle-voix crédible. Nous avons mesuré combien l'adaptation de la voix lyrique au cinéma, et notamment cette technique du play-back, ont une incidence directe sur le traitement du corps et de la voix de l'interprète, combien elle contraint les chanteurs habitués à la scène à un profond un travail de métamorphose physique, scénique et vocale. Celle-ci s'opère principalement dans un souci de crédibilité à l'écran, sinon de précision. De la même façon, chaque cinéaste envisage le play-back différemment, pour montrer l'exercice du chant à l'écran avec plus ou moins de véracité. Du point de vue de la critique, ce recours à la dissociation corps-voix demeure sujet à polémique, aussi bien à cause des problématiques liées à l'adéquation physique entre interprète et personnage, qu'en raison des carences irrémédiables du play-back, qui ne saurait égaler la qualité du son direct. Néanmoins, nous pouvons nous demander si les avancées techniques en matière de prise de son et de post-production ne permettraient pas aujourd'hui une meilleure gestion du play-back et une plus grande qualité d'enregistrement, et si ces progrès suffiraient à pallier ces problèmes, à satisfaire davantage la critique et à réhabiliter ce genre très controversé. Déjà, pour la Tosca de Benoît Jacquot, film le plus récent du corpus datant des années 2000, la critique s'est montrée bien plus élogieuse à l'égard de la qualité de la bande-son, ce qui n'est certainement pas étranger aux progrès techniques. Nous ne pouvons nous prononcer pour ce film sur une éventuelle amélioration de la synchronisation, puisque justement, le réalisateur n'a pas cherché, pour des raisons esthétiques précédemment évoquées, la perfection ni le réalisme du play-back. En revanche, la presse a souligné la qualité de la scénarisation acoustique et la précision des effets de spatialisation de cette Tosca ${ }^{47}$. De même, elle lui a reconnu une meilleure correspondance ente perspective sonore et perspective visuelle, entre acoustique musicale et acoustique cinématographique ${ }^{48}$, là où les films plus anciens présentaient encore d'importantes lacunes.

\section{BIBLIOGRAPHIE}

\section{Ouvrages}

CIMENT Michel et ROSI Francesco, Le dossier Rosi, Paris, Plon, 1987.

SACCO Laure-Hélène, «L'opéra à l'épreuve du cinéma », thèse de doctorat, Université Sorbonne Nouvelle - Paris 3, 2012.

SALIERI Frantz, " Don Juan Raimondi », in Don Giovanni. Livret de Lorenzo Da Ponte et autres documents, Gaumont Vidéo, 2005.

\section{Articles}

Sur Don Giovanni, de Joseph Losey

DAVID Catherine, « Monsieur Losey saisi par l'opéra », Le Nouvel Observateur, 19.11.1979. 
DOUCELIN Jacques, « Rolf Liebermann : "Réduire le pouvoir du réalisateur" ", Le Figaro, 23.02.1983.

DRILLON Jacques et LAFON François, « Ruggero Raimondi : "Losey a fait de moi un acteur" », Le Monde de la musique, 01.10.1979.

FLEURET Maurice, « Don Juan dans tous ses états », Le Nouvel observateur, 05.11.1979.

MOUTON Michel, « Ils sont trois : il y a Mozart, il y a Palladio, et il y a Losey... », Le Monde de la musique, 01.10.1979.

SACCO Laure-Hélène, « Le Don Giovanni de Mozart vu par Joseph Losey », in Don Juan, Le Paon d'Héra / Hera's Peacock n7, Éditions du Murmure, 2010, 364 p. (Issn : 978-2-915099-41-3)

Sur Carmen, de Francesco Rosi

CHALAIS François, « Une tragédie andalouse », Le Figaro Magazine, 17.03.1984.

LOISEAU Jean-Claude, « Rosi : Carmen me ressemble », Le Point, 12.03.1984.

MÉRIGAUD Bernard, « Carmen. La reine soleil... entre deux barbons », Télérama, 17.03.1984.

MONTAIGNE Pierre, « Carmen en majesté », Le Figaro, 29.06.1983.

REY Anne, « Les ambiguïtés du film-opéra », Le Monde, 30.05.1986.

SACCO Laure-Hélène, "Musique, chant et danse dans la Carmen de Francesco Rosi », in Musique, chant et danse en Europe latine et Amérique Latine, Université Paris III, 2011, http://www.univparis3.fr/publications-de-l-ed-122-18730.kjsp?RH=1232622291102

TRANCHANT Marie-Noëlle, « Un œil noir... nous regarde », Le Figaro, 14.03.1984.

VILLIEN Bruno, « Francesco Rosi face à Carmen », Diapason, 01.04.1984.

Sur La Bohème, de Luigi Comencini

HENDRICKS Barbara, « Barbara Hendricks. Mimì la perle d'ébène. », Le Républicain Lorrain, 14.03.1988.

LAFON François, « Barbara Hendricks, le don du naturel », Le Monde de la musique, 01.12.1987.

PASCAUD Fabienne, « Mimì passion », Télérama, 09.03.1988.

SARAZIN Michel, « Les malheurs de Mimì la cousette », Le Journal du dimanche, 26.07.1987.

SICLIER Jacques, « Un décor état d'âme », Le Monde, 05.08.1987.

VILLIEN Bruno, « La Bohème à l'écran », Opéra International, 01.10.1987.

Sur Tosca, de Benoît Jacquot

BONNAUD Frédéric, « Scandale », Les Inrockuptibles, 13.11.2001.

FRODON Jean-Michel et Roux Marie-Aude, « Benoît Jacquot, réalisateur de Tosca », Le Monde, 14.11.2001.

HEYMANN Danièle, « Parfait opéra », Marianne, 03.12.2001.

LOISEAU Jean-Claude, «Entretien avec Benoît Jacquot, réalisateur de Tosca », Télérama, 17.11.2001.

MÉRIGAUD Bernard, « Un opéra magnifié par la caméra », Télérama, 17.11.2001.

ROUX Marie-Aude, « Du disque à la B.O., cinq minutes de décalage... », Le Monde, 14.11.2001.

Sur d'autres films d'opéra 
LOMPECH Alain, « De l'art difficile du jardin japonais », Le Monde, 23.11.1995.

MÉRIGEAU Pascal, « Puccini du côté de chez Frédéric Mitterrand », Le Monde, 23.11.1995.

SACCO Laure-Hélène, «L'opéra à l'épreuve de l'image mobile : le film d'opéra », in Musique et Cinéma : harmonies et contrepoints, Inter-Lignes, Laboratoire Art, Culture et Transmission, Revue de laFaculté des Lettres et des Sciences Humaines de Toulouse, 2010.

Filmograhie :

COMENCINI Luigi, La Bohème, [vidéo], Erato film [prod.], UGC [distrib.], 1988.

JACQUOT Benoît, Tosca, [DVD], Paris, TF1 vidéo [éd.], 2002.

LOSEY Joseph, Don Giovanni, [DVD], Neuilly-sur-Seine, Gaumont vidéo éditions, 2006.

ROSI Francesco, Carmen, [DVD], Édition Collector, Neuilly-sur-Seine, Gaumont vidéo éditions / Boulogne-Billancourt, Paramount Home Entertainment France, 2010 (version remasterisée).

\section{NOTES}

1. Michel Sarazin, « Les malheurs de Mimì la cousette ", Le Journal du dimanche, 26 juillet 1987.

2. Francesco Rosi, propos recueillis par Bruno Villien, « Francesco Rosi face à Carmen », Diapason, 01 avril 1984, p. 40.

3. Jacques Siclier, «Un décor état d'âme », Le Monde, 05 août 1987.

4. Frantz Salieri, "Don Juan Raimondi", dans Don Giovanni. Livret de Lorenzo Da Ponte et autres documents, Gaumont Vidéo, 2005, p. 37. (Élément constitutif du coffret DVD Don Giovanni MozartLosey, Gaumont DVD, 2006.)

5. Ibid.

6. Frantz Salieri, Ibid., p. 39.

7. Michel Sarazin, « Les malheurs de Mimì la cousette », op. cit.

8. Francesco Rosi, propos recueillis par Jean-Claude Loiseau, « Rosi : Carmen me ressemble ", Le Point, 12 mars 1984, p. 122.

9. Ibid.

10. Julia Migenes-Johnson, propos recueillis par Marie-Noëlle Tranchant, « Un œil noir... nous regarde ", Le Figaro, 14 mars 1984.

11. Joseph Losey, propos recueillis par Catherine David, «Monsieur Losey saisi par l'opéra », Le Nouvel Observateur, 19 novembre 1979, p. 134.

12. Rolf Liebermann, propos recueillis par Jacques Doucelin, «Rolf Liebermann: "Réduire le pouvoir du réalisateur" ", Le Figaro, 23 février 1983.

13. Anne Rey, «Les ambiguïtés du film-opéra », Le Monde, 30 mai 1986.

14. Barbara Hendricks, propos recueillis par Fabienne Pascaud, «Mimì passion ", Télérama, 09 mars 1988.

15. Ibid.

16. Ruggero Raimondi, propos recueillis par Jacques Drillon et François Lafon, «Ruggero Raimondi : "Losey a fait de moi un acteur" ", Le Monde de la musique, 01 Octobre 1979, p. 112.

17. Ibid.

18. Ibid.

19. Janine Reiss, propos recueillis par Michel Mouton, «Ils sont trois », Le Monde de la musique, 01 octobre 1979, p. 27.

20. Luca Canonici, propos recueillis par Bruno Villien, « La Bohème à l'écran », Opéra International, 01 octobre 1987. 
21. Barbara Hendricks, «Barbara Hendricks. Mimì la perle d'ébène. », Le Républicain Lorrain, 14 mars 1988.

22. Ruggero Raimondi, propos recueillis par Jacques Drillon et François Lafon, «Ruggero Raimondi : "Losey a fait de moi un acteur" ", op. cit., p. 111.

23. Joseph Losey, propos recueillis par Catherine David, "Monsieur Losey saisi par l'opéra », op. cit.

24. Frantz Salieri, « Don Juan Raimondi », op. cit.

25. Ibid.

26. Ibid.

27. Joseph Losey, « Notes de Joseph Losey ", dans Don Giovanni. Livret de Lorenzo Da Ponte et autres documents, op. cit., p. 28.

28. Francesco Rosi, propos recueillis par Michel Ciment, in Le dossier Rosi, Paris, Plon, 1987, p. 265.

29. Ibid.

30. Ibid.

31. Julia Migenes-Johnson, propos recueillis par Pierre Montaigne, "Carmen en majesté », Le Figaro, 29 juin 1983.

32. Barbara Hendricks, propos recueillis par François Lafon, "Barbara Hendricks, le don du naturel », Le Monde de la musique, 01 décembre 1987, p. 20.

33. Benoît Jacquot, propos recueillis par Jean-Claude Loiseau, «Entretien avec Benoît Jacquot, réalisateur de Tosca », Télérama, 17 novembre 2001.

34. Ibid.

35. Benoît Jacquot, propos recueillis par Jean-Michel Frodon et Marie-Aude Roux, «Benoît Jacquot, réalisateur de Tosca », Le Monde, 14 novembre 2001.

36. Ibid.

37. Barbara Hendricks, propos recueillis par François Lafon, «Barbara Hendricks, le don du naturel », op. cit.

38. Benoît Jacquot, propos recueillis par Jean-Claude Loiseau, « Entretien avec Benoît Jacquot, réalisateur de Tosca », op. cit.

39. Ibid.

40. Alain Lompech, « De l'art difficile du jardin japonais », Le Monde, 23 novembre 1995.

41. François Chalais, « Une tragédie andalouse », Le Figaro Magazine, 17 mars 1984.

42. Ibid.

43. Par exemple : Pierre Murat, "Carmen. La reine soleil... entre deux barbons ", Télérama, 17 mars 1984.

44. Pascal Mérigeau, "Puccini du côté de chez Frédéric Mitterrand», Le Monde, 23 novembre 1995.

45. Danièle Heymann, « Parfait opéra », Marianne, 03 décembre 2001.

46. Frédéric Bonnaud, « Scandale », Les Inrockuptibles, 13 novembre 2001.

47. Bernard Mérigaud, « Un opéra magnifié par la caméra », Télérama, 17 novembre 2001.

Marie-Aude Roux, «Du disque à la B.O., cinq minutes de décalage... », Le Monde, 14 novembre 2001.

48. Maurice Fleuret, «Don Juan dans tous ses états ", Le Nouvel observateur, 05 novembre 1979 : «Un opéra est fait pour l'acoustique uniforme du théâtre, alors que le cinéma implique de changer d'acoustique presque aussi fréquemment qu'on change de plan. Si la résonnance est modifiée ou seulement modulée en cours de phrase, voire d'une scène à l'autre, c'est toute la continuité musicale qui en souffre. Si elle ne l'est pas, c'est l'image qui perd de sa vérité avec un son qui, tout à coup, paraît venir d'ailleurs ". 


\section{RÉSUMÉS}

Cette étude s'intéresse à la question du passage à l'écran de la voix lyrique, et à la problématique qu'il soulève sur le plan du rapport voix-corps. Dans le cadre d'une adaptation cinématographique d'un opéra, comment appréhender la voix chantée, comment filmer le corps chantant, tout en conciliant les exigences du cinéma et celles de l'opéra ? La réflexion évalue la difficulté de faire coïncider, tant esthétiquement que techniquement, le corps et la voix. Elle traite la question du play-back et du doublage ainsi que l'enjeu de ce dédoublement entre la voix et le corps sur les plans de la synchronisation mouvements-voix, et de la vraisemblance de la gestuelle des interprètes. Il s'agit également de mesurer l'impact de ce passage sur grand écran sur le travail vocal, corporel et scénique des chanteurs, destiné à s'adapter à l'écriture cinématographique. À l'issue de cette analyse est abordée la question de la réception, à travers une étude sur la façon dont ont été perçus par la critique ces choix relatifs au rapport voix-corps dans le film d'opéra.

This study takes an interest in the question on how the lyric voice is heard on the screen, and how it raises the issue in terms of voice-body connection. As part of a film adaptation of an opera, how to grasp the singing voice, how to shoot the singing body, while balancing the demands of the film and those of the opera? Reflection assesses the difficulty to match, both aesthetically and technically, body and voice. It addresses the issue of playback and dubbing as well as the challenge of this splitting between voice and body in terms of synchronizing movements and voice, and the likelihood of gesture interpreters. It is also to measure the impact of this transition on the big screen about voice work, body work and singers stage work, designed for being adapted to film writing. As the outcome of this analysis the question of reception is discussed, through a study on how these critical choices about the voice-body ratio were perceived by in the opera film.

\section{INDEX}

Mots-clés : Film d'opéra, Voix lyrique au cinéma , Rapport corps-voix, Play-back, Synchronisation, Adéquation physique et vocale entre interprète et personnage, Réception

\section{AUTEUR}

\section{LAURE-HÉLÈNE SACCO}

Docteur en études italiennes de l'Université Paris 3, qualifiée aux fonctions de maître de conférences en section 18 (études cinématographiques et études théâtrales) et en section 14 (études italiennes), a été ATER à la Sorbonne Nouvelle et à l'Université d'Avignon. Elle est notamment l'auteur d'une thèse de doctorat intitulée « L'opéra à l'épreuve du cinéma ", soutenue en octobre 2012 et effectuée sous la direction de Madame Myriam Tanant. 